\title{
MASS TRANSFER WITH COMPLEX REVERSIBLE CHEMICAL REACTIONS-I. SINGLE REVERSIBLE CHEMICAL REACTION
}

\author{
G. F. VERSTEEG, J. A. M. KUIPERS, F. P. H. VAN BECKUM \\ and W. P. M. VAN SWAAIJ \\ Department of Chemical Engineering, Twente University of Technology, P.O. Box 217, 7500 AE \\ Enschede, Netherlands
}

(First received 8 December 1986; owing to external reasons accepted in revised form 3 February 1989)

\begin{abstract}
An improved numerical technique was used in order to develop an absorption model with which it is possible to calculate rapidly absorption rates for the phenomenon of mass transfer accompanied by a complex reversible chemical reaction. This model can be applied for the calculation of the mass transfer rates (and enhancement factors) for a wide range of processes and conditions, for both film model and penetration model, complex kinetic expressions and equilibrium reactions. With the aid of this method it is demonstrated that reversibility has a substantial effect on the absorption rate. Approximate analytical solutions for the calculation of the mass transfer rates presented in literature are checked for their validity. All approximations are of restricted use and can be applied only for a limited number of reactions and it is desirable to check the approximation with the aid of a numerical solution before it is used for mass transfer calculations. The linearization method of Hikita and Asai (Kagaku Kogaku 11, 823-830, 1963) cannot be applied generally for reversible reactions and therefore can lead to erroneous results. Experimentally determined absorption rates of $\mathrm{H}_{2} \mathrm{~S}$ and $\mathrm{CO}_{2}$ in various aqueous alkanolamine solutions can be predicted satisfactorily for the several mass transfer regimes studied.
\end{abstract}

\section{INTRODUCTION}

In the process industry, operations involving mass transfer followed by reversible chemical reactions occur very frequently. Well known examples are the amine gas-treating processes where the acid gas components $\mathrm{H}_{2} \mathrm{~S}$ and/or $\mathrm{CO}_{2}$ are consumed by a chemical reaction in the alkanolamine solution during the absorption and subsequently released by the reverse reaction during the regeneration of the rich solvent.

For design purposes it is extremely important that models are available which can be used for the calculation of the mass transfer rates for the various process conditions. Therefore the theoretical modelling of the phenomenon mass transfer followed by a chemical reaction has been studied intensively for the various types of mass transfer models, for instance the film theory and penetration theory. However, analytical solutions could be obtained for a few very special cases only. For the film theory Olander (1960) gave an analytical solution for the problem of mass transfer with a reversible chemical reaction which could be regarded as instantaneous with respect to mass transfer. Also analytical solutions for both film and penetration theory have been presented for first-order reversible and irreversible reactions (Sherwood and Pigford, 1952; Danckwerts and Kennedy, 1954; Huang and Kuo, 1965). For all other situations, with reactions different from the cases mentioned above, no analytical solution could be obtained and therefore numerical techniques must be used in solving these phenomena.

An alternative approach in finding a method for the calculation of the mass transfer rate, i.e. the enhancement factor, is by approximation and/or lineariz- ation. This was first introduced by van Krevelen and Hoftijzer (1948), who treated the reaction terms and approximated the concentration profiles, and so a solution for the film model could be obtained for mass transfer followed by a second-order bi-molecular irreversible chemical reaction. This method was extended for $(m, n)$ th-order irreversible chemical reaction by Hikita and Asai (1963) and, similarly, the surface renewal model for second-order irreversible chemical reactions was solved by DeCoursey (1974). In case reversible reactions occur, an approximate solution for the film model was developed by Onda et al. (1970), who linearized the reactions similar to the method proposed by Hikita and Asai (1963), and by Huang and Kuo (1965), but the solution of Huang is of restricted use. Onda et al. (1972) also developed in the same way a solution for the penetration model and DeCoursey (1982) developed the surface renewal description but in both cases the diffusivity of all species have to be equal. Hikita et al. (1982) derived an approximate solution for the penetration model but for this approach it is necessary that the solute loading is close to zero. Although the restriction of equal diffusivities for all species was not imposed on this solution, the applicability seems to be very restricted because of the zero loading condition.

Numerically solved descriptions of the mass tansfer rate for systems with mass transfer followed by a reversible reaction were also presented (Perry and Pigford, 1953; Secor and Beutler, 1967; Cornelisse et al., 1980). They all applied the finite-difference solution method on the set of differential equations of the penetration theory. The work of Perry and Pigford (1953) can be regarded as a first introduction in 
calculating mass transfer rates numerically. The other publications are of restricted use because a limited range of conditions has been studied and the influence of solute loading and the behaviour under desorption conditions has been omitted (Secor and Beutler, 1967), or the applicability has been restricted to a few stoichiometric schemes (Cornelisse et al., 1980). DeCoursey (1982) questioned the solutions of Secor and Beutler for the special case where the chemical equilibrium constant was equal to one. Moreover, Secor and Beutler presented no results of calculations of enhancement factors above a value of eight which may lead to the conclusion that probably for some of the solutions presented numerical problems occurred.

In this paper a numerical method is presented for the calculation of mass transfer rates for the phenomenon mass transfer followed by a complex reversible chemical reaction for both. film and penetration theory. The applied numerical technique was fundamentally the same as the method used by Cornelisse et al. (1980). However, several refinements of this technique were introduced to increase the accuracy and minimise the computational time. In order to obtain a generally applicable model the kinetics of the reactions considered were of a general form and could be varied casily. Furthermore the approximate solutions, which were mentioned before, were verified and the boundaries of the validity will be presented. Finally, the presented model is tested experimentally for the absorption of $\mathrm{H}_{2} \mathrm{~S}$ or $\mathrm{CO}_{2}$ into several aqueous amine solutions in a model reactor (stirred vessel).

In near future this model will be extended to the case of several parallel liquid-phase reactions and to the case of the simultaneous absorption of two gasphase components in the liquid phase where also several parallel reactions occur.

\section{THEORY}

\subsection{Introduction}

The problem considered is mass transfer followed by a reversible chemical reaction of general order with respect to both reactants and products:

$$
A(g)+\gamma_{b} B(1) \leftrightarrows \gamma_{c} C(l)+\gamma_{d} D(l)
$$

with the following reaction rate equation:

$$
\begin{aligned}
R_{a}= & k_{m, n, p, q}[A]^{m}[B]^{n}[C]^{\rho}[D]^{q} \\
& -k_{r, s, t, v}[A]^{r}[B]^{s}[C]^{\prime}[D]^{v}
\end{aligned}
$$

Other expressions for the reaction rate like for instance Langmuir-Hinschelwood kinetics and the zwitterion-mechanism for the reaction between $\mathrm{CO}_{2}$ and alkanolamines (Blauwhoff et al., 1984) can easily be included in the model developed in the present study. However, most reactions for gas-liquid systems can be expressed sufficiently accurate with a reaction rate expression similar to eq. (2) and therefore this expression was used in this model.

The mass transfer in the gas phase was described with the stagnant film model while for the liquid phase both the stagnant film and the penetration model were used. For the liquid phase both models were applied because these two can be considered as the two extremes of all theoretical models developed as far as concerning the influence of the diffusivity. Furthermore the penetration model is expected to be the most realistic one in gas-liquid absorption in stirred vessels to be discussed later on (Versteeg et al., 1987).

\subsection{Penetration model}

For the penetration model the material balances for each species for the phenomenon mass transfer followed by a chemical reaction yields the following set of equations:

$$
\begin{aligned}
& \frac{\partial[A]}{\partial t}=D_{a} \frac{\partial^{2}[A]}{\partial x^{2}}-R_{a} \\
& \frac{\partial[B]}{\partial t}=D_{b} \frac{\partial^{2}[B]}{\partial x^{2}}-\gamma_{b} R_{a} \\
& \frac{\partial[C]}{\partial t}=D_{c} \frac{\partial^{2}[C]}{\partial x^{2}}+\gamma_{c} R_{a} \\
& \frac{\partial[D]}{\partial t}=D_{d} \frac{\partial^{2}[D]}{\partial x^{2}}+\gamma_{d} R_{a} .
\end{aligned}
$$

These four non-linear partial differential equations must be solved numerically because an analytical solution method is not available. Before solving this set of equations uniquely, one initial condition and two boundary conditions are necessary. As initial condition it is assumed that the system considered is in equilibrium for a given solute loading:

$$
\begin{aligned}
t=0 \text { and } x \geqslant 0,[A]= & {[A]_{0},[B]=[B]_{0}, } \\
& {[C]=[C]_{0},[D]=[D]_{0} }
\end{aligned}
$$

where the concentrations $[A]_{0},[B]_{0},[C]_{0}$ and $[D]_{0}$ satisfy $R_{a}=0$ in eq. (2). The boundary condition for $x$ $=\infty$ can also be derived with the assumption of chemical equilibrium for the bulk of the liquid for a given solute loading:

$$
\begin{aligned}
t>0 \text { and } x=\infty,[A]= & {[A]_{0},[B]=[B]_{0}, } \\
& {[C]=-[C]_{0},[D]-[D]_{0} . }
\end{aligned}
$$

In case of irreversible chemical reactions $(K=\infty)$ eq. (8) can be used only for situations where $[A]_{0}=0$ (or $[B]=0$ which is identical to physical absorption), because for concentrations of $A$ different from zero the liquid bulk is not in equilibrium and a reaction will occur. In the latter case boundary condition (8) is not valid and a material balance over the liquid bulk must be used instead. Note that the application of eqs (7) and ( 8$)$ is only valid if the equilibrium composition is used.

The second boundary condition is obtained by assuming that the species $B, C$ and $D$ are non-volatile and that the flux of component $A$ in the gas phase is equal to the flux in the liquid phase. The use of the latter assumption instead of $[A]=[A]_{2}$ at $x=0$ is convenient in view of the applicability of the model for absorber calculations especially for those cases where a part of 
the resistance against mass transfer is situated in the gas phase:

$$
\begin{gathered}
k_{g}\left([A]_{g, \text { bulk }}-[A]_{g, i}\right)=-D_{a}\left(\frac{\partial[A]}{\partial x}\right)_{x=0} \\
\left(\frac{\partial[B]}{\partial x}\right)_{x=0}=\left(\frac{\partial[C]}{\partial x}\right)_{x=0}=\left(\frac{\partial[D]}{\partial x}\right)_{x=0}=0
\end{gathered}
$$

\subsection{Infinite reaction rate constants}

In case of infinite reaction rate constants the boundary condition (9) is no longer valid and the gradients of $[B],[C]$ and $[D]$ are not necessarily zero in order to take into account that these components are nonvolatile.

Io derive the appropriate boundary conditions one may return to the mass balances over a small layer $\Delta x$ adjacent to the gas-liquid interface, depicted in Fig. 1. For the four components one finds:

$$
\begin{aligned}
& \frac{\partial}{\partial t} \int_{0}^{\Delta x}[A] \mathrm{d} x=J_{a}+D_{a}\left(\frac{\partial[A]}{\partial x}\right)-\int_{0}^{\Delta x} R_{a} \mathrm{~d} x \\
& \frac{\partial}{\partial t} \int_{0}^{\Delta x}[B] \mathrm{d} x=D_{b}\left(\frac{\partial[B]}{\partial x}\right)-\int_{0}^{\Delta x} \gamma_{b} R_{a} \mathrm{~d} x \\
& \frac{\partial}{\partial t} \int_{0}^{\Delta x}[C] \mathrm{d} x=D_{c}\left(\frac{\partial[C]}{\partial x}\right)+\int_{0}^{\Delta x} \gamma_{c} R_{a} \mathrm{~d} x \\
& \frac{\partial}{\partial t} \int_{0}^{\Delta x}[D] \mathrm{d} x=D_{d}\left(\frac{\partial[D]}{\partial x}\right)+\int_{\theta}^{\Delta x} \gamma_{d} R_{a} \mathrm{~d} x .
\end{aligned}
$$

For finite reaction rate constants and finite time derivatives all integrals vanish when $\Delta x$ approaches zero and eq. (9) is recovered. In the limit situation that the reaction rate constants have infinite values, the reaction rate, $R_{\alpha}$, becomes indefinite and therefore the integrals are also indefinite. The way to proceed is first to eliminate $R_{\mathrm{a}}$ by adding and subtracting among the eqs (10) and then to take the limit that $\Delta x \rightarrow 0$, by which the accumulation terms vanish under the assumption that the time derivatives of all concen-

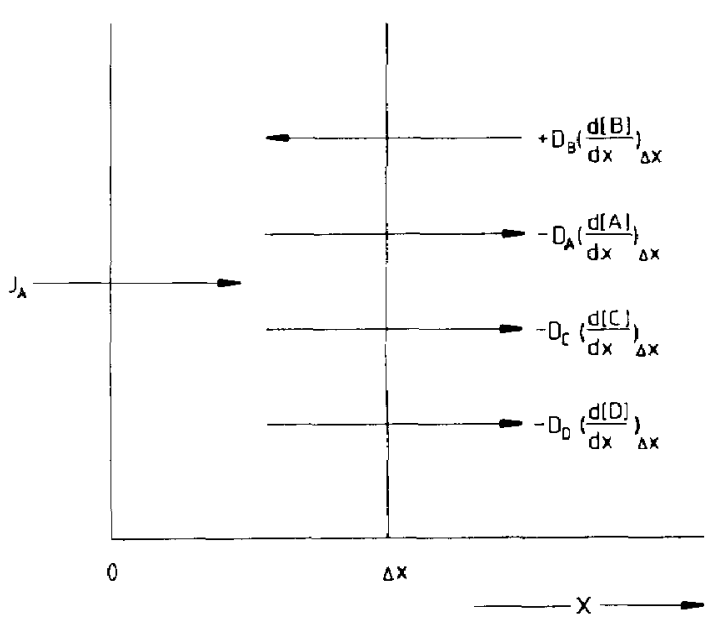

Fig. 1. Fluxes at the interface. trations remain bounded. In this way one finds (for the situation that all stoichiometric coefficients are equal):

$$
\begin{gathered}
J_{a}+D_{a}\left(\frac{\partial[A]}{\partial x}\right)_{x=0}+D_{c}\left(\frac{\partial[C]}{\partial x}\right)_{x=0}=0 \\
D_{b}\left(\frac{\partial[B]}{\partial x}\right)_{x=0}+D_{c}\left(\frac{\partial[C]}{\partial x}\right)_{x=0}=0 \\
D_{b}\left(\frac{\partial[B]}{\partial x}\right)_{x=0}+D_{d}\left(\frac{\partial[D]}{\partial x}\right)_{x=0}=0 .
\end{gathered}
$$

This elimination process gives three independent eqs (11), therefore the fourth equation to achieve the complete boundary conditions has to express the value of the quantity $R_{a}$ which just has been eliminated. In case of infinite reaction rate constants and with the assumption that at the gas-liquid interface equilibrium is maintained this fourth equation will be the equilibrium condition.

In view of applicability it is desirable to obtain one general solution for the phenomenon mass transfer followed by a reversible chemical reaction. Therefore the set of eqs (3), (4), (5) and (6) will be solved with boundary condition (9). For the limit situation where the reaction rate constants become infinite, the description will be approximated by extremely high values of $k_{m, n, p, q}$ and $k_{r, s, t, v}$. Afterwards this approximation has to be verified which is possible with the aid of the analytical solution of Olander $(1960)$. In case that for the description of the phenomenon mass transfer accompanied by complex chemical reaction besides the eqs (3), (4), (5) and (6) also equilibrium equations are used (Cornelisse et al., 1980) boundary condition (11) must be used instead of condition (9).

\subsection{Film model}

For the film model the phenomenon mass transfer followed by a chemical reaction can be represented with the following set of equations:

$$
\begin{array}{r}
D_{a} \frac{\partial^{2}[A]}{\partial x^{2}}-R_{a}=0 \\
D_{b} \frac{\partial^{2}[B]}{\partial x^{2}}-\gamma_{b} R_{a}=0 \\
D_{c} \frac{\partial^{2}[C]}{\partial x^{2}}+\gamma_{c} R_{a}=0 \\
D_{d} \frac{\partial^{2}[D]}{\partial x^{2}}+\gamma_{d} R_{a}=0
\end{array}
$$

and the following boundary conditions:

$$
\begin{aligned}
& \text { at } x=0: k_{g}\left([A]_{g, \text { bulk }}-[A]_{g, i}\right)=-D_{a}\left(\frac{\partial[A]}{\partial x}\right)_{\substack{x=0 \\
(12 a}} \\
& \left(\frac{\partial[B]}{\partial x}\right)_{x=0}=\left(\frac{\partial[C]}{\partial x}\right)_{x=0}=\left(\frac{\partial[D]}{\partial x}\right)_{x=0}=0 . \\
& \text { at } x=\delta:[A]=[A]_{0},[B]=[B]_{0},
\end{aligned}
$$

$$
[C]=[C]_{0},[D]=[D]_{0} \text {. }
$$


For infinite reaction rate constants the boundary conditions (11) derived in Section 2.3 should be used.

\subsection{Enhancement factor}

The enhancement factor, $E_{a}$, defined as the ratio of the mass flux of component $A$ through the interface with chemical reaction and driving force $\left([A]_{i}-[A]_{0}\right)$ to the mass flux through the interface without chemical reaction, but with the same driving force, can be obtained for both models from the calculated concentration profiles. The enhancement factor, $E_{a}$, is defined by:

-penetration model:

$k_{l} E_{a}\left([A]_{i}-[A]_{0}\right)=\left(\frac{1}{\theta}\right) \int_{0}^{\theta}-D_{a}\left(\frac{\mathrm{d}[A]}{\mathrm{d} x}\right)_{x=0} \mathrm{~d} t$

film model:

$$
k_{l} E_{a}\left([A]_{i}-[A]_{0}\right)=-D_{a}\left(\frac{\mathrm{d}[A]}{\mathrm{d} x}\right)_{x=0}
$$

However, these concentration profiles are calculated with boundary conditions $(9 a)$ and (12a) for the penetration model and the film model respectively and therefore the value of the numerically calculated enhancement factor, as defined above, has only a physical meaning in those situations where the gas-phase mass transfer resistance can be neglected completely (i.e. $[A]_{g, 0}=[A]_{g, i}$ ).

\section{NUMERICAL TREATMENT}

\subsection{Introduction}

In the film model the main goal of a numerical approach is to produce the concentration profiles as solution of a system of coupled non-linear ordinary differential equations with two point boundary conditions. From the concentration profiles obtained after solving this set of equations the mass transfer rate (e.g. the enhancement factor) can be calculated easily. From the numerical point of view the film model can be regarded as a special case of the penetration model. Therefore the numerical technique used for solving these differential equations will not be discussed separately.

In the penetration (Higbie) model the concentration profiles are time-dependent: they develop as solution of a system of coupled non-linear parabolic partial differential equations subject to specified initial and two point boundary conditions. The approach used to solve these models is mainly based on the method presented by Cornelisse et al. (1980), however, some new numerical features are introduced in order to increase the accuracy and minimise the computational time.

\subsection{Discretization}

The discretization itself follows mainly the lines by Cornelisse et at. (1980). Therefore only a few additional remarks are presented.

The distribution of spatial grid points in the $x, t$ - plane can be manipulated by defining a (sufficiently differentiable) transformation function $x=\Phi(z)$. In order to move the grid points towards the interface the derivative of $\Phi$ near $z=0$ should be small compared with elsewhere. So an uniform mesh size in $z$ now automatically generates a higher concentration of grid points near the interface (see Fig. 2).

The same can be done with respect to the time variable, $t$. Also here the grid spacing near the origin is refined, as the steepest time-derivatives occur for small $t$, being induced by the concentration jump between gas and liquid at the start of the contact period.

An example for the spatial (place) transformation is:

$$
x=p z+(1-p) z^{4}
$$

with parameter $p$ between 0 and 1 , controlling the grid deformation. For $p=1$ the identity $x=z$ is obtained and for lower $p$-values the grid deformation increases. [It should be avoided that $p=0$, in order to retain the one-to-one correspondence between the derivatives $(\mathrm{d} c / \mathrm{d} x)$ and $(\mathrm{d} c / \mathrm{d} z)$ given by the formula $(\mathrm{d} c / \mathrm{d} z)$ $=(\mathrm{d} c / \mathrm{d} x) \cdot(\mathrm{d} x / \mathrm{d} z)$.]

For the time coordinate a quadratic transformation:

$$
t-w^{2}
$$

is a suitable choice. A typical grid in the $x, t$-plane is depicted in Fig. 3 and from this figure it can be seen that near the interface (small $x$ ) and at the start (short $t$ ) the concentration of grid points is higher than elsewhere. Discretizing (partial) differential equations in a transformed coordinate system is somewhat more complicated then in the original variables because of the transformation derivatives that occur in the differential operators. For instance, transforming the second $x$-derivative in terms of $z, z$ being given by $x$ $=\Phi(z)$, leads to:

$$
\frac{\partial^{2}[A]}{\partial x^{2}}=\frac{1}{\phi^{\prime}(z)} \frac{\partial}{\partial z}\left(\frac{1}{\phi^{\prime}(z)} \frac{\partial[A]}{\partial z}\right)
$$

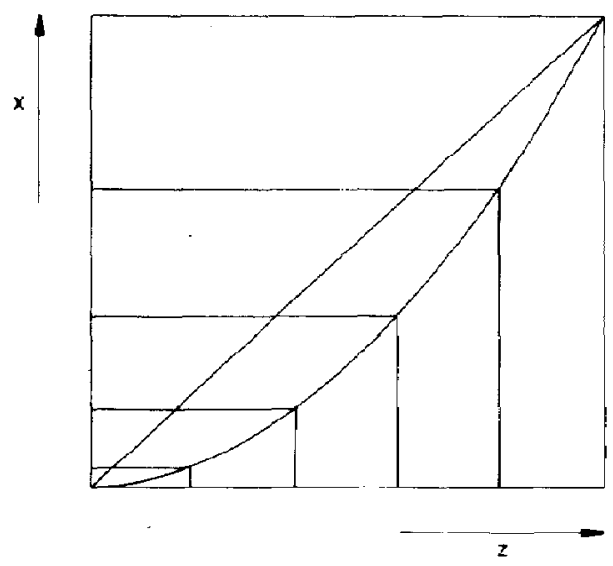

Fig. 2. Effect of transformation on the spatial variable. 


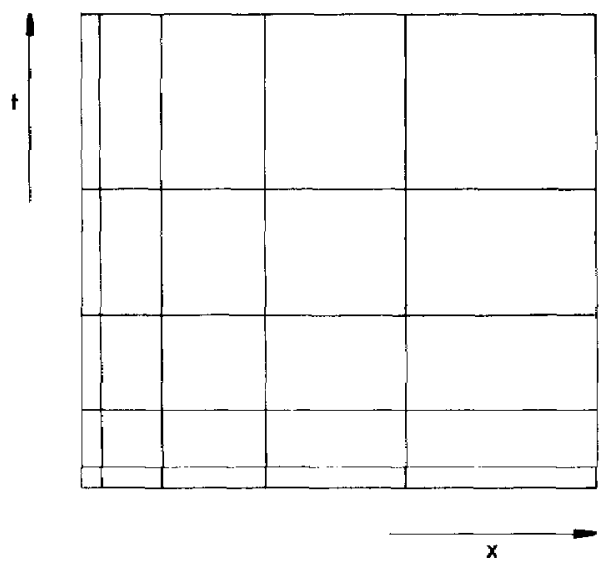

Fig. 3. A typical grid in the $x, t$-plane.

where $\Phi^{\prime}$ is the derivative of $\Phi(z)$, which is known analytically. Now in a point $z_{\mathrm{m}}(=m \Delta z)$, on a grid with uniform mesh size $\Delta z$ and subscript $m$ indicating the place level, the discretization of eq. (18) becomes:

$$
\begin{aligned}
\frac{1}{\phi^{\prime}\left(z_{m}\right)} \frac{1}{\Delta z}\left(\frac{1}{\phi^{\prime}\left(z_{m+0.5}\right)} \frac{[A]_{m+1}-[A]_{m}}{\Delta z}\right. \\
\left.-\frac{1}{\phi^{\prime}\left(z_{m-0.5}\right)} \frac{[A]_{m}-[A]_{m-1}}{\Delta z}\right) .
\end{aligned}
$$

For the time derivative a three-point backward discretization is used (with superscript $j$ indicating the time level) leading to:

$$
\frac{\partial[A]}{\partial t} \rightarrow \frac{3[A]_{m}^{j+1}-4[A]_{m}^{j}+[A]_{m}^{j-1}}{2 \Delta t} .
$$

The finite difference form of the penetration (Higbie) model thus leads to relations between concentrations in five grid points, clustered as a "molecule" shown in Fig. 4. Only for $j=0$ this molecule is impossible, because grid points with time index -1 do not exist. Therefore in the first step a two-point backward discretization (Euler) is used leading to:

$$
\frac{\partial[A]}{\partial t} \rightarrow \frac{[A]_{m}^{1}-[A]_{m}^{0}}{\Lambda t}
$$

at the cost of a lower order truncation error in this time step.

\subsection{Special transformation}

In the present approach a special transformation of the independent variables has been introduced taken from the analytical solution of the well known onedimensional diffusion problem:

$$
\begin{array}{rlrl}
\frac{\partial[A]}{\partial t}=D_{a} \frac{\partial^{2}[A]}{\partial x^{2}} & \\
t=0 \text { and } x \geqslant 0 & {[A]} & =[A]_{0} \\
t>0 \text { and } x=0 & {[A]} & =[A]_{i} \\
t>0 \text { and } x=\infty & {[A]} & =[A]_{0} .
\end{array}
$$
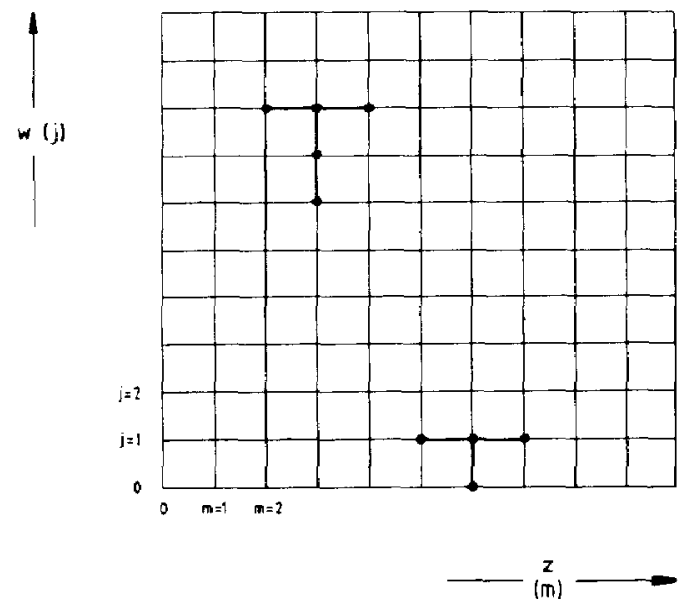

Fig. 4. Discretization scheme.

The solution of eq. (22) is given by:

$$
\frac{[A]-[A]_{0}}{[A]_{i}-[A]_{0}}=\operatorname{errc}\left(\frac{x}{\sqrt{4 D_{a} t}}\right)
$$

Concentration profiles according to eq. (24) are shown in Fig. 5, illustrating the well known phenomenon of penetration depth. At any time, say $t^{*}$, the significant behaviour of the concentration profile is restricted within an interval $0 \leqslant x \leqslant x^{*}$, where $x^{*}$ is a function of $t^{*}$. In view of eq. (24) this function between $x^{*}$ and $t^{*}$ is chosen usually as:

$$
x^{*}=\text { constant } \sqrt{4 D_{a} t^{*}}
$$

with a value of the constant depending on what one is inclined to call "significant behaviour".

Now in numerical calculations it looks worthwhile to restrict the calculational domain to the interval given by the penetration depth, and to spend no grid points on the remaining part of the $x$-axis where almost nothing occurs. This adaptive grid point distribution is most easily accomplished if a coordinatetransformation according to eq. (26) is employed:

$$
\begin{aligned}
& r=\operatorname{erf}\left(\frac{x}{\sqrt{4 D_{s} t}}\right) \\
& \tau=\sqrt{\frac{t}{\theta}} .
\end{aligned}
$$

In Fig. 6 the effect of the transformation is illustrated starting with a uniform grid in $r$, $\tau$-space. For short contact times the spacing is very fine, leading to a high concentration of grid points and so to a high resolution near the spot where the sharpest gradients occur.

Besides the above mentioned feature, several additional advantageous properties concerning the numerical treatment are introduced with the transformation of the independent variables as specified by eq. (26). Firstly, the serni-infinite $x$-domain is mapped in the $r$-domain on the finite interval $[0,1]$. Secondly, 


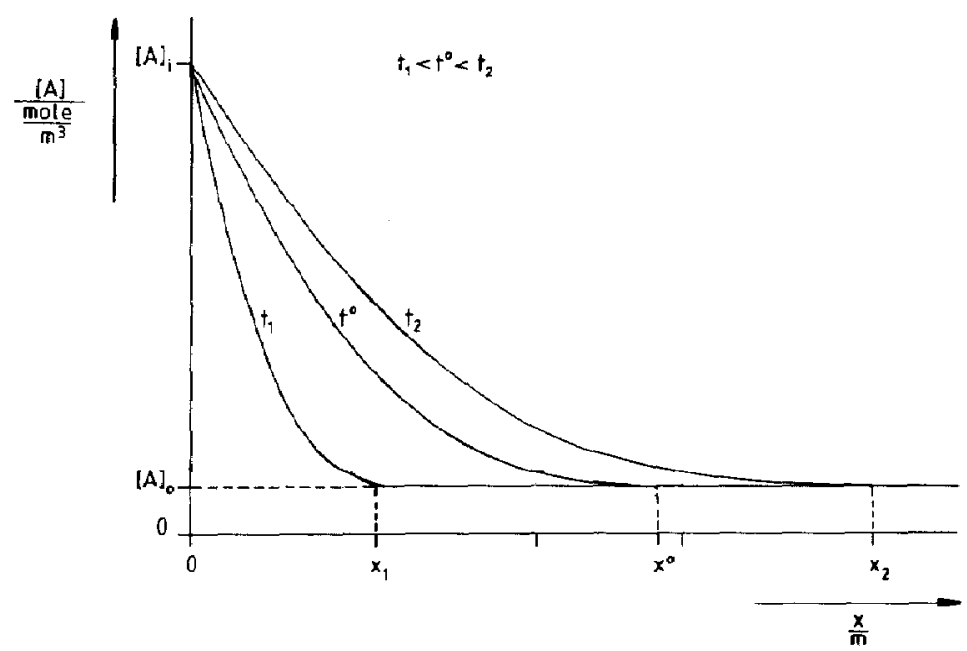

Fig. 5. Concentration profile for the physical absorption for several contact times.

in the $r, \tau$-domain the $r$-derivative of the concentration eq. (24) at the gas-liquid interface is bounded when $\tau$ approaches the value zero whereas the $x$-derivative is unbounded for $t=0$ :

$$
\begin{gathered}
x, t \text {-domain: }\left(\frac{\partial[A]}{\partial x}\right)_{x=0}=\frac{[A]_{i}}{\sqrt{\pi D_{a} t}} \\
r, \tau \text {-domain: }\left(\frac{\partial[A]}{\partial r}\right)_{r=0}=[A]_{i} .
\end{gathered}
$$

Finite domains and finite derivatives are highly desirable if not necessary for reliable numerical results. When applying this transformation on the system of four coupled partial differential equations [eqs (3), (4), (5) and (6)], the maximum diffusion coefficient, $D_{m}$, of the components involved should be taken in eq. (25) in order to assure that this component will not run out of the picture. The equation for this component, say $A$, becomes:

$$
\tau \frac{\partial[A]}{\partial \tau}=\frac{2}{\pi} \exp \left(-2 \xi(r)^{2}\right) \frac{\partial^{2}[A]}{\partial r^{2}}-2 \tau^{2} \theta R_{a}
$$

while the equations for the other components are affected with more complicated expressions, e.g. for component $C$ :

$$
\begin{aligned}
\tau \frac{\partial[C]}{\partial \tau}= & \frac{2}{\pi} d_{i} \exp \left[-\left(\frac{1}{d_{i}}+1\right)(\xi(r))^{2}\right] \\
& \times \frac{\partial}{\partial r}\left(\exp \left[\left(\frac{1}{d_{i}}-1\right)(\xi(r))^{2}\right] \frac{\partial[C]}{\partial r}\right) \\
& +2 \tau^{2} \theta \gamma_{c} R_{c}
\end{aligned}
$$

with:

$$
\zeta(r)=\text { inverse } \operatorname{erf}\{r\}
$$

and

$$
d_{\mathrm{i}}=\frac{D_{i}}{D_{m}}
$$

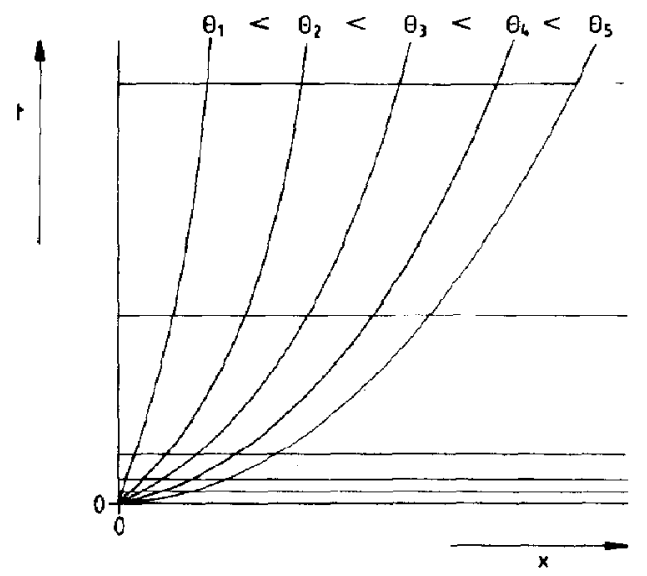

Fig. 6. Fffect of transformation according to eq. (26) on the spatial and time variable. 
The boundary conditions ( 8 ) are now located at $r=1$ :

$$
\begin{aligned}
\tau>0, r=1:[A]=[A]_{0},[B] & =[B]_{0}, \\
{[C] } & =[C]_{0},[D]=[D]_{0}
\end{aligned}
$$

and the boundary condition (9) at the interface becomes:

$$
\begin{gathered}
\tau>0, r=0: \frac{\partial[A]}{\partial r}=-\sqrt{\pi D_{m} \tau} \frac{k_{g}}{D_{a}}\left([A]_{g, \text { bulk }}\right. \\
\left.-[A]_{g, i}\right) \\
\left(\frac{\partial[B]}{\partial r}\right)_{r=0}=\left(\frac{\partial[C]}{\partial r}\right)_{r=0}=\left(\frac{\partial[D]}{\partial r}\right)_{r=0}=0 .
\end{gathered}
$$

It is interesting to note that this transformation has given the physical phenomena a chronological order. Under the assumption that $R_{a}$ and all the derivatives of concentrations with respect to $\tau$ and $r$ are bounded, the terms in eq. (28) can be distinguished by the powers of $\tau$.

The diffusion operator is independent of $\tau$ and for $\tau=0$ it is the only term present in the differential equation. Then for $\tau>0$ the accumulation term, which is linear in $\tau$, becomes important because it has to take into account the incoming flux generated by the boundary condition (32a), which by itself is coming up also linearly in $\tau$. This is the phenomenon of purely physical absorption.

Finally, with increasing $\tau$, the reaction term becomes effective, it acts on the concentration profiles built up by the physical absorption and is trying to turn them back to equilibrium values, which exist already at the boundary $r=1$ [see eq. (31)]. So, in fact, after the physical absorption, coming in from the gas-liquid interface $(r=0)$, the reaction term is a kind of response from the liquid bulk. Being quadratic in $\tau$ this effect will predominate in the end, pressing the concentration profiles back to the left and filling up the whole interval with the equilibrium values.

So for $t \rightarrow \infty$ this coordinate system $\{r, \tau\}$ may become inadequate, but it is advantageous in the beginning, when the most rapid changes occur, due to the sudden onset of the interface condition (Ya). Especially the Newton-Raphson linearization of the non-linear reaction term, eq. (2), using estimates of a previous time level may be awkward just because of the large changes from one time level to another. In this new coordinate system, however, the onset of the interface conditions is not abrupt but gradual, starting from zero and rising linearly in $\tau$, so in the interior all the differences will be gradual. Accordingly the estimates in the linearization of the reaction term will be much more accurate. Besides, the reaction term itself is small, of order $\tau^{2}$.

\subsection{Remarks on the initial condition}

The boundary conditions for the $r, \tau$-description are easily transferred from the original problem, the interface $(x=0)$ coincides with $r=0$ and the bulk $(x=\infty)$ with $r=1$.
The initial conditions, however, require special attention because of the topological consequences of the transformation. In view of eq. (26) the initial line $(t=0$ and $x>0$ ) is mapped upon one point $\tau=0, r=1$, so the initial condition (7) in the $x, t$-plane is moved to that one point:

$$
\begin{aligned}
\tau=0, r=1:[A]=[A]_{0},[B] & =[B]_{0}, \\
{[C] } & =[C]_{0},[D]=[D]_{0} .
\end{aligned}
$$

In the meanwhile the origin in the $x, t$-plane seems to be stretched upon the whole interval $\tau=0,0<r<1$, and it is not obvious beforehand what kind of initial condition is originating from this point.

The answer to this question is a differential problem itself, consisting of ordinary differential equations along the $r$-axis, together with two point boundary conditions. It is the differential problem that turns up when the limit $\tau \rightarrow 0$ is taken in the partial differential problem [eq. (28)]. In the differential equations only the diffusion operators mentioned earlier are retained [see eq. (28)]:

and

$$
\frac{\partial^{2}[A]}{\partial r^{2}}=0
$$

$$
\begin{array}{r}
\frac{\partial}{\partial r}\left[d _ { i } \operatorname { e x p } [ - ( \frac { 1 } { d _ { i } } + 1 ) ( \xi ( r ) ) ^ { 2 } ] \frac { \partial } { \partial r } \left[\exp \left(\frac{1}{d_{i}}-1\right)\right.\right. \\
\left.\times\left((\xi(r))^{2} \frac{\partial[C]}{\partial r}\right)\right]=0
\end{array}
$$

and also for the components $B$ and $D$. The boundary conditions at $r=0$ follow from eq. (32) for $\tau \rightarrow 0$ :

$$
\begin{array}{r}
\tau=0, \quad r=0:\left(\frac{\partial[A]}{\partial r}\right)_{r=0}=\left(\frac{\partial[B]}{\partial r}\right)_{r=0}=\left(\frac{\partial[C]}{\partial r}\right)_{r=0} \\
=\left(\frac{\partial[D]}{\partial r}\right)_{r=0}
\end{array}
$$

while the boundary conditions at $r=1$ where already given by eq. (31), which again is the limit of eq. (33) for $\tau \rightarrow 0$. The solution of the differential problem eqs (33), (34) and (35) is:

$$
\begin{array}{r}
\tau=0,0<r<1: \quad[A]=[A]_{0},[B]=[B]_{0},[C]=[C]_{0} \\
\text { and }[D]=[D]_{0}
\end{array}
$$

and therefore this is the initial condition in the $r$, $\tau$-plane.

In this case the $r, t$-initial condition eq. (36) is quite similar to the $x, t$-initial-condition eq. (7). However, this section is meant to warn the reader not to copy it from eq. (7) but to realise the consequences of the transformation. In fact the model problem [eqs (22) and (23)] is a non-trivial example. In the $r, \tau$-planc the formulation is:

$$
\begin{aligned}
\frac{\partial[A]}{\partial \tau} & =\frac{2}{\pi} \exp \left(-2 \xi(r)^{2}\right) \frac{\partial^{2}[A]}{\partial r^{2}} \\
\tau>0 & \text { and } r=0:[A]=[A]_{i} \\
\tau>0 & \text { and } r=1:[A]=[A]_{0}
\end{aligned}
$$


while the initial condition is found from the ordinary differential problem:

$$
\begin{gathered}
\frac{\partial^{2}[A]}{\partial r^{2}}=0 \\
r=0:[A]=[A]_{i} \\
r=1:[A]=[A]_{0} .
\end{gathered}
$$

The solution of this differential problem is:

$$
\tau=0,0<r<1: \quad[A]=[A]_{i}-\left([A]_{i}-[A]_{0}\right)^{*} r
$$

This initial condition is clearly quite different from eq. (23a). [In this case the expression (37d) satisfies eq. (37a) and so it is identical with the solution of the partial differential problem (37), given by eq. (24).]

\section{APPROXIMATE SOLUTIONS}

\subsection{DeCoursey approach}

DeCoursey (1982) derived an approximate analytical solution for the set of eqs (3), (4), (5) and (6) with initial and boundary conditions (7), (8) and (9). $\mathrm{He}$ obtained this solution by changing the instantaneous concentrations to time mean concentrations according to the Danckwerts' surface renewal model by taking "s-multiplied" Laplace transforms. In order to obtain an integrable set linear differential equations with constant coefficients he made the following assumptions:

- the diffusivities of all species are equal,

- the equilibrium at any point can be expressed by $[A]_{e}=[C][D] /(K[B])$,

$-[B]$ is equal to $[B]_{i}$ near the interface according to van Krevelen and Hoftijzer (1948),

-the reaction rate can be described with $R_{a}=K_{1}$ $\left([A]-[A]_{0}\right)+f_{1}(x)$,

- the reaction rate at $x=\infty$ can be expressed by $R_{a}$ $=k_{2}[B]_{i}\left([A]-[A]_{e}\right)=0$ and at $x=0$ by $R_{a}$ $=k_{2}[B]_{i}\left([A]-[A]_{e i}\right)$.

At $t>0$ and $x=\infty$ the concentrations of $A, B, C$ and $D$ are in equilibrium according to:

$$
K=\frac{[C][D]}{[A][B]} .
$$

The enhancement factor can be calculated according to DeCoursey's set of eqs (33), (34) and (35).

This approximate solution was checked in that work for three limit situations, $K=\infty$ and $[A]_{0}=0$, $R_{a}=\infty$, and $M \approx 0$, and the agreement with the ana1ytical solutions was extremely good.

\subsection{Onda approach}

Onda et al. $(1970,1972)$ presented approximate solutions for both film model and penetration model. For the film model the set of eqs (3a), (3b), (3c) and (3d) were solved with boundary conditions (12) and (13) and for the penetration model the set of eqs (3), (4), (5) and (6) with initial and boundary conditions (7), (8) and (9). In order to obtain an analytical solution for this set of equation, he linearized the reaction rate expressions according to the method proposed by Hikita and Asai (1963) leading to:

$$
\begin{aligned}
{[B]=} & {[B]_{i} \text { for } 0 \leqslant x \leqslant \delta } \\
{[F]=} & {[F]_{i} \text { for } 0 \leqslant x \leqslant \delta } \\
R_{a}= & \frac{2}{m+1} \gamma_{a} k_{m, n}[A]_{i}^{m-1}[B]_{i}^{n}[A] \\
& -\frac{2}{p+1} \gamma_{a} k_{p, q}[E]_{i}^{p-1}[F]_{i}^{q}[E] .
\end{aligned}
$$

The enhancement factor can be calculated with his eqs (9), (11), (12), (21) and (22) but it should be noted that for the trial and error method the enhancement factor, $E_{a}$, should be taken as iteration parameter instead of $e_{i}$, as was proposed originally by the author, in order to obtain a stable solution method over a wide range of conditions. However, the enhancement factor calculated in this way is not comparable with the enhancement factor defined according to eq. (14) or (15) and the enhancement factor calculated according to the Onda approximation should be multiplied with $[A]_{i} /\left([A]_{i}-[A]_{0}\right)$. The approximate solution of the penetration model has the additional restriction that the diffusivity of all species must have the same value.

It should be noted also that Onda treated the reaction products $E$ and $F$ in a different way in the linearization step which led to his somewhat strange eq. (19) in which the expression for the equilibrium constant is a function of the reaction orders $m$ and $p$. Furthermore, the reaction rate expression, $R_{a}$, should be zero at $x=\delta$ indicating that in the liquid bulk the equilibrium composition is maintained. For the linearized rate expression used by Onda, $R_{a}$ can have values different from zero at $x=\delta$ and only for the very special case that $[A]_{0}=\lceil E\rceil_{0}=0$ is this condition always fulfilled.

The approximate solutions has been compared in that work with numerical solutions; however, this has been restricted to situations with $[A]_{0}=0$ and low values of $E_{\mathrm{a}}$.

\subsection{Hikita approach}

Hikita et al. (1982) presented an approximate solution for the penetration model and for the reaction

$$
A+B \leftrightarrows C+D
$$

according to the set of eqs (3), (4), (5) and (6) with initial and boundary conditions (7), (8) and (9). The following assumptions have been made:

-the concentrations of the species $B, C$ and $D$ in the liquid near the gas-liquid interface were assumed to be constant and equal to their interfacial concentrations and independent of the exposure time of the liquid to the gas (Hikita and Asai, 1963),

- the chemical equilibrium has been established 
according to:

$$
K=k_{2} / k_{-2}=\left([C]_{0}[D]_{0}\right) /\left([A]_{0}[B]_{0}\right) .
$$

The enhancement factor can be estimated with their implicit eq. (11) for the situation that $[A]_{0}=[C]_{0}$ $=[D]_{0}=0$. This latter condition implies that this approximation can be applied only to situations where the conversion of the reactants is very low.

Hikita's approximate solution was well in line with the numerical solution obtained by Secor and Beutler (1967), the maximum deviation being about $6 \%$.

\section{RESULTS}

\subsection{Numerical results}

In order to check the influence of the analytical transformation according to eq. (26), runs of the numerical solution method with and without transformation (26) respectively have been carried out on three examples. Both numerical solutions are also compared with the analytical solutions for the same three runs to check the validity of the numerical method used and to investigate the accuracy of the solutions. The two numerical methods applied are:

(A) transformations (16) and (17):

- fix the bulk values on a finite $z$, far enough to be of no influence on the results

- number of $w$-steps (time grid) equal to the number of $z$-steps (spatial grid) equal to $N$

- take $p=0.01$, eq. (16)

(B) transformation (26), followed by transformations (16) and (17):

-take equal number of $w$-steps and $z$-steps $(=N)$,

-take $p=0.01$.

The examples are:

(1) physical absorption: $A(g) \rightarrow A(l)$,

(2) absorption and first order irreversible chemical reaction:

$$
\begin{aligned}
& A(g) \rightarrow A(l) \\
& A(l) \rightarrow B(l) \text { with } R_{a}=k_{1}[A]
\end{aligned}
$$

(3) absorption and bimolecular irreversible chemical reaction and "infinite" reaction rate constant, $k_{2}$ :

$$
\begin{aligned}
& A(g) \rightarrow A(l) \\
& A(l)+2 B(l) \rightarrow P(l) \text { with } R_{a}=k_{2}[A][B] .
\end{aligned}
$$

As an illustration of the performance the calculated enhancement factors are presented in Table 1.

From Table 1 it can be concluded that with the implementation of an additional transformation according to eq. (26) the accuracy of the numerical solutions can be improved drastically, even at a small number of grid points. Especially for highly reactive gas-liquid systems (e.g. example 3) transformation (26) is an extremely powerful method to minimise the computational time necessary to calculate the mass transfer rate and the enhancement factor. For example 3 , the decrease of the required computational time due to the new method is roughly about a factor 9 , for equal accuracy of the calculated enhancement factor, as it can be derived from Table 1 .

The differences between analytical and numerical solutions are very small (see Table 1) and therefore with the numerical technique applied in the present study it is possible to calculate the mass transfer rate for the phenomenon mass transfer accompanied by reversible chemical reactions.

The boundary condition defined by eq. (9) is not valid for those situations where the reaction can be regarded as an equilibrium reaction with infinite reaction rate constants, indicating that equilibrium is maintained at all places in the liquid, and so the numerical model used, strictly spoken, cannot be applied for this type of reactions. However, in order to check the applicability of the model, the equilibrium reactions are approximated by means of extremely high reaction rate constants and this approximation is verified by means of a comparison with the analytical solution presented by Secor and Beutler (1967) for the film model. In Table 2 the results are presented of this comparison for a reaction [see eq. (1)] with all stoichiometric coefficients equal to one $\left(\gamma_{a}=\gamma_{b}=\gamma_{c}=\gamma_{d}\right.$ $=1$ ) and both forward and reverse reaction overall second order respectively. From Table 2 it can be concluded that for equilibrium reactions the mass transfer rate (and the enhancement factor) can be

\begin{tabular}{|c|c|c|c|c|c|c|}
\hline \multirow[b]{2}{*}{$N$} & \multicolumn{2}{|c|}{ Example 1} & \multicolumn{2}{|c|}{ Example 2} & \multicolumn{2}{|c|}{ Example 3} \\
\hline & $A$ & $B$ & $A$ & B & $A$ & $B$ \\
\hline 20 & 0.9906 & 1.0075 & 101.078 & 100.675 & 636.14 & 141.85 \\
\hline 40 & 0.9927 & 1.0013 & 101.203 & 100.096 & 152.81 & 142.58 \\
\hline 60 & 0.9948 & 1.0005 & 100.082 & 100.033 & 141.95 & 142.80 \\
\hline 80 & 0.9960 & 1.0003 & 100.044 & 100.017 & 140.99 & 142.82 \\
\hline \multicolumn{3}{|c|}{$\begin{array}{l}\text { Exact value of } \quad 1.0000 \\
\text { analytical solution }\end{array}$} & \multicolumn{2}{|c|}{100.004} & \multicolumn{2}{|c|}{142.82} \\
\hline
\end{tabular}
calculated with satisfactory accuracy with the approximation applied.

The influence of the reversibility of the reaction on

Table 1. Comparison of numerical solutions with analytical solutions 
Table 2. Comparison of numerical solutions with analytical solutions for "infinite" reaction rate constants

\begin{tabular}{lcrrrrr}
\hline & \multicolumn{2}{c}{$K_{e}=10^{-3}$} & \multicolumn{2}{c}{$K_{e}=10^{0}$} & \multicolumn{2}{c}{$K_{e}=10^{3}$} \\
$\alpha$ & $E_{\text {num }}$ & $E_{\text {an }}$ & $E_{\text {num }}$ & $E_{\text {an }}$ & $E_{\text {num }}$ & \multicolumn{1}{c}{$E_{\text {ar }}$} \\
\hline $10^{-3}$ & 1.264 & 1.264 & 10.42 & 10.41 & 92.54 & 92.51 \\
$10^{-2}$ & 1.170 & 1.170 & 9.61 & 9.60 & 91.64 & 91.61 \\
$10^{-1}$ & 1.078 & 1.078 & 5.64 & 5.64 & 82.73 & 82.70 \\
0.50 & 1.040 & 1.039 & 2.52 & 2.52 & 44.86 & 44.84 \\
0.80 & 1.032 & 1.032 & 2.01 & 2.01 & 18.35 & 18.34 \\
0.99 & 1.029 & 1.029 & 1.83 & 1.83 & 4.02 & 4.00 \\
\hline
\end{tabular}

Physical constants: $D_{a}=D_{b}=D_{c}=D_{d}=1 \times 10^{-9} \mathrm{~m}^{2} \mathrm{~s}^{-1} . \quad H e=1 \mathrm{~mol}$ $\mathrm{mol}^{-1}$

Concentrations: $[B]_{t}=1000 \mathrm{~mol} \mathrm{~m}{ }^{-3} \cdot[A]_{i}=10 \mathrm{~mol} \mathrm{~m}^{-3}$.

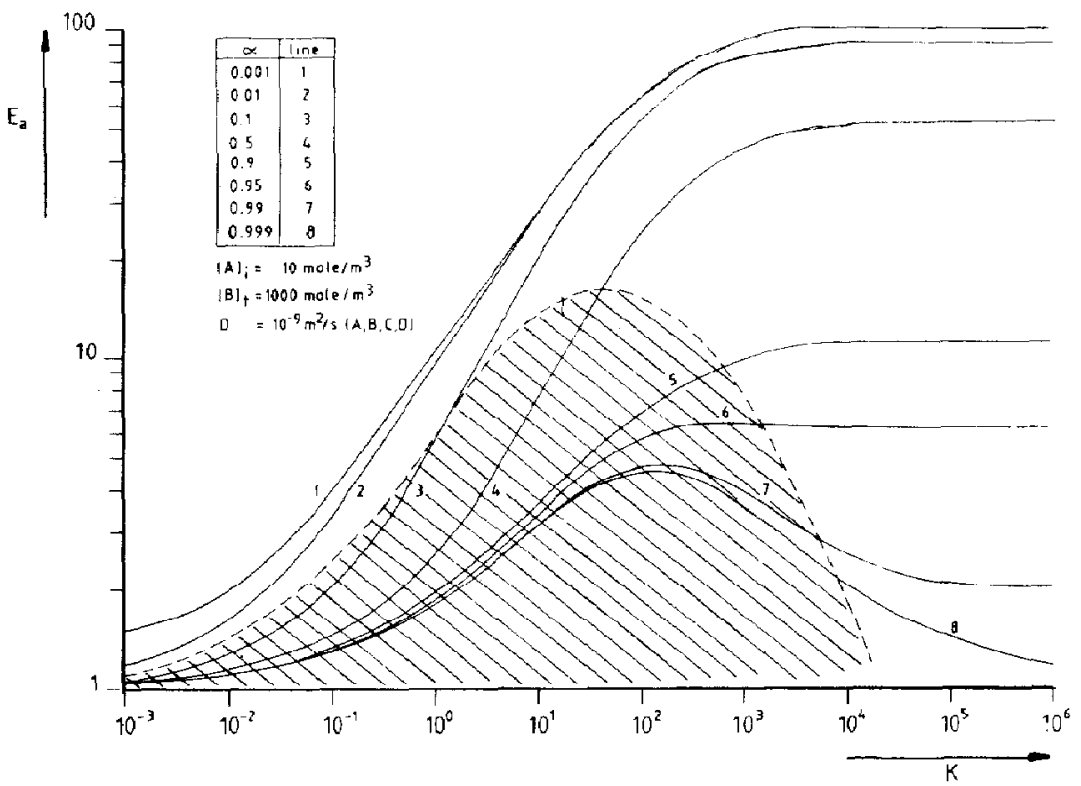

Fig. 7. Enhancement factor as function of the equilibrium constant, $K$, for various solute loadings. The dashed line represents the conditions where no net mass transfer occurs, under this line desorption prevails.

the enhancement factor is demonstrated in the Figs 7 and 8 for $k_{g} \approx \infty$. In Fig. 7 the enhancement factors according to the film model, the results for the penetration model are similar, are presented as a function of the chemical equilibrium constant for several solute loadings for the reaction:

$$
A+B \leftrightarrows C+D
$$

with "infinite" reaction rate constants and reaction rate equation:

$$
R_{a}=k_{1,1,0,0}[A][B]-k_{0,0,1,1}[C][D] .
$$

For high values of $K\left(=k_{1,1,0,0} / k_{0,0,1,1}\right)$ this situation is identical to absorption followed by an instantaneous irreversible reaction with respect to mass transfer and the enhancement factor, $E_{a}$, can be calculated for this particular reaction with the analytically derived expression for this regime:

$$
E_{a}=1+\frac{D_{b}[B]_{o}}{D_{a}[A]_{i}} .
$$

From Fig. 7 it can be concluded that the enhancement factor increases with the equilibrium constant because the reverse reaction rate constant decreases and therefore the effect of the reverse reaction. However, with increasing solute loadings the enhancement factor shows a maximum and decreases for higher values of the equilibrium constant. For these conditions, high solute loading and high equilibrium constant, the bulk concentration of $B$ further decreases for higher equilibrium constants. After the equilibrium composition of the liquid remains constant the enhancement factor can be calculated with eq. (39). In Fig. 7 two regimes are shown, the dashed area represents the conditions where desorption prevails and the area above the absorption regime. At the boundary of the two areas no net mass transfer occurs. A similar plot was 


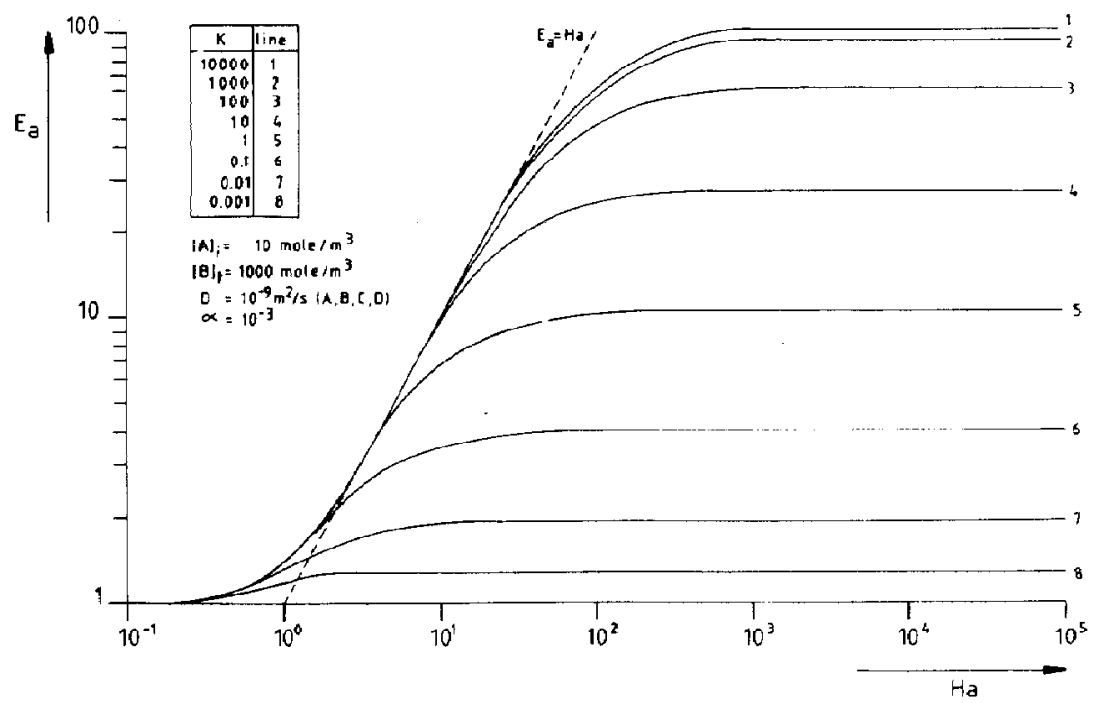

Fig. 8. Enhancement factor as function of the $\mathrm{Ha}$-number for various equilibrium constants.

produced by Cornelisse et al. (1980) for the penetration model but due to the lower solvent loadings used in their calculations no optimum in the enhancement factor was found.

In Fig. 8 the enhancement factors according to the penetration model, the results according to the film model are similar, are presented as function of the $\mathrm{Ha}$ number for several equilibrium constants and a relatively low solute loading, $\alpha=10^{-3}$, for the same reaction as mentioned above. In Fig. 8 can be seen that the reversibility also has a pronounced effect on the enhancement factor for reactions with finite reaction rate constants. It should be noted that the deviation from the line $E_{a}=H a$, indicating that diffusion limitation of component $B$ occurs, is starting at lower Hatta-numbers with decreasing equilibrium constant. This effect is extremely important for the determination of the reaction kinetics of reactions with small equilibrium constants by means of absorption experiments and the assumption of pseudo first order reaction conditions (see Danckwerts, 1970), because it is certainly possible that this latter assumption is not fulfilled, even for low solute loadings, although the Hatta-number is greater than 2 and much smaller than $E_{a, \infty}$ calculated according to eq. (39). Therefore the influence of reversibility on the mass transfer rates should always be studied if the kinetics of a reaction are to be determined by means of absorption measurements in order to avoid erroneous results.

\subsection{DeCoursey approach}

The DeCoursey approximate solution was checked with the numerical solution of the Higbie penetration theory although this approximate solution was derived for the surface renewal theory. The difference between both theoretical models e.g. for a first order irreversible reaction is always less than $2 \%$. The reaction considered was:

$$
A+B \leftrightarrows C+D
$$

with reaction rate equation:

$$
R_{a}=k_{1,1,0,0}[A][B]-k_{0,0,1,1}[C][D]
$$

and the conditions used for the comparison with the present numerical solutions are summarized in Table 3. The deviation with the numerical solutions is always less than $10 \%$.

With the DeCoursey approximation the enhancement factor can be calculated accurately for the surface renewal model over a wide range of process conditions. However, the condition that the diffusivities of all species considered must have equal values is a substantial restriction for the applicability of this approximation. Nevertheless, the linearization of the reaction equation developed by DeCoursey seems to be a good alternative for the method of Hikita and Asai (1963).

\subsection{Onda approach}

The approximate solutions of Onda were checked with the present numerical solutions for four different reaction types (film model) and three types (penetration theory) respectively as is summarized in Table 4 , and Table 5 gives the process conditions studied.

Table 3. Conditions for the comparison of the DeCoursey approximation

\begin{tabular}{ll}
\hline Diffusivity (all species) & $10{ }^{9} \mathrm{~m}^{2} \mathrm{~s}^{-1}$ \\
{$[B]_{t}$} & $1000 \mathrm{~mol} \mathrm{~m}^{-3}$ \\
{$[A]_{i}$} & $10 \mathrm{~mol} \mathrm{~m} \mathrm{~m}^{-3}$ \\
Equilibrium constant & $10^{-3} \leqslant K \leqslant 10^{4}$ \\
Solute loading & $10^{-3} \leqslant \alpha \leqslant 0.999$ \\
Ha-number & $0.10 \leqslant H a \leqslant 10^{6}$
\end{tabular}


Table 4. Reaction types for the comparison of the Onda approximation with the corresponding numerical solutions

\begin{tabular}{clll}
\hline $\begin{array}{l}\text { Film model } \\
\text { Type 1 }\end{array}$ & $A \leftrightarrow 2 E$ & & \\
Type 2 & $A+B \leftrightarrow C+D$ & $R_{\mathrm{a}}=k_{1}[A]-k_{2}[E]^{2}$ & $\mathrm{~mol} \mathrm{~m}^{-3} \mathrm{~s}^{-1}$ \\
Type 3 & $A+2 B \leftrightarrow C+D$ & $R_{a}=k_{2}[A][B]-k_{2}[C][D]$ & $\mathrm{mol} \mathrm{m}^{-3} \mathrm{~s}^{-1}$ \\
Type 4 & $A+2 B \leftrightarrow 2 C+D$ & $R_{a}=k_{3}[A][B]^{2}-k_{-2}[C][D]$ & $\mathrm{mol} \mathrm{m}^{-3} \mathrm{~s}^{-1}$ \\
& & $R_{a}=k_{3}[A][B]^{2}-k_{3}[C]^{2}[D]$ & $\mathrm{mol} \mathrm{m}^{-3} \mathrm{~s}^{-1}$ \\
Penetration model & & \\
Type 1 & $A+B \leftrightarrow C+D$ & $R_{a}=k_{2}[A][B]-k_{2}[C][D]$ & $\mathrm{mol} \mathrm{m}^{-3} \mathrm{~s}^{-1}$ \\
Type 2 & $A+2 B \leftrightarrow C+D$ & $R_{a}=k_{3}[A][B]^{2}-k_{-2}[C][D]$ & $\mathrm{mol} \mathrm{m}^{-3} \mathrm{~s}^{-1}$ \\
Type 3 & $A+2 B \leftrightarrow 2 C+D$ & $R_{a}=k_{3}[A][B]^{2}-k_{-3}[C]^{2}[D]$ & $\mathrm{mol} \mathrm{m}^{-3} \mathrm{~s}^{-1}$ \\
\hline
\end{tabular}

Table 5. Conditions for the comparison of the Onda approximation

\begin{tabular}{|c|c|}
\hline $\begin{array}{l}\text { Diffusivity (all species) } \\
{[B]_{t}} \\
{[A]_{i}} \\
\text { Equilibrium constant } \\
\text { Ha-number }\end{array}$ & $\begin{array}{l}10^{-9} \mathrm{~m}^{2} \mathrm{~s}^{-1} \\
1000 \mathrm{~mol} \mathrm{~m}^{-3} \\
10 \mathrm{molm} \mathrm{m}^{-3} \\
10^{-3} \leqslant K \leqslant 10^{4} \\
0.10 \leqslant H a \leqslant 10^{6}\end{array}$ \\
\hline $\begin{array}{ll}\text { Solute loading } & \\
\text { Film model: } & \text { type 1 } \\
& \text { type } 2 \\
& \text { type } 3 \\
& \text { type } 4\end{array}$ & $\begin{array}{l}10^{-3} \leqslant \alpha \leqslant 0.999 \\
10^{-3} \leqslant \alpha \leqslant 0.999 \\
10^{-3} \leqslant \alpha \leqslant 0.499 \\
10^{-3} \leqslant \alpha \leqslant 0.499\end{array}$ \\
\hline $\begin{array}{r}\text { Penetration model: } \\
\text { type } 1 \\
\text { type } 2 \\
\text { type } 3\end{array}$ & $\begin{array}{l}10^{-3} \leqslant \alpha \leqslant 0.999 \\
10^{3} \leqslant \alpha \leqslant 0.499 \\
10^{-3} \leqslant \alpha \leqslant 0.499\end{array}$ \\
\hline
\end{tabular}

From the comparison of reaction types 1,2 and 3 for the film model it could be concluded that the maximum deviation from the numerical model is always less than $2 \%$. However, for reaction type 4 the Onda approximation is not able to obtain realistic values for the enhancement factor (i.e. $\geqslant 1$ ) in case the reverse reaction can not be neglected (i.e. solute loadings which substantially differ from zero and/or low equilibrium constants). This is a remarkable result and may be caused by the linearization of the reaction term by Onda where the two reaction products are treated in a different way (and led to the somewhat strange expression for the equilibrium constant, Onda's relation (19) (Onda et al., 1970)). This may result in an overestimation of the reverse reaction and can lead to a net reverse reaction according to Onda's reaction rate eq. (13) (Onda et al., 1970) although in view of the equilibrium a net forward reaction should occur or an overall reaction rate equal to zero. Therefore the Onda approximation for the film model can only be applied satisfactorily if the values for $m$ and $t$ are the same and if $p=q=r=s=0$ in the reaction rate expression [eq. (2) 7. In case only one product is formed, e.g. reaction type 1, this approximation can be applied satisfactorily without the restriction that $m=t$.

For the penetration theory approximation the same conclusions are relevant and the maximum deviation for both reaction types 1 and 2 is always less than $7 \%$. It should be noted that this approximation can only be used in case all the diffusivities are the same which restricts its applicability.

From the comparison of the outcome of the calculations for the Onda approach with the numerical solutions it can be concluded that the linearization of the reaction term according to Hikita and Asai (1963) cannot be applied uniformly to reversible reactions and that Onda's eq. (13) (Onda et al., 1970) is not able to describe correctly all possible types of reactions due to the fact that the products were treated in different ways in the linearization of the reaction rate expression.

\subsection{Hikita approach}

This approximation was studied for reaction type 1 only (see Table 4, penetration theory) and the process conditions are summarized in Table 6 . The maximum deviation with numerical solutions was always less than $6 \%$. Although the diffusivities of the various components need not to be equal as in the Onda and DeCoursey approach, the applicability is severely restricted because the solute loading should be virtually zero. Moreover, as the same linearization technique as Onda et al. (1970) was used it is likely that an extension of this approach to solute loadings different from zero will not lead to a more general applicable approximation.

Table 6. Conditions for the comparison of the Hikita approximation

\begin{tabular}{ll}
\hline Diffusivity component $A$ & $10^{-9} \mathrm{~m}^{2} \mathrm{~s}^{-1} \leqslant D_{a} \leqslant 2 \times 10^{-9} \mathrm{~m}^{2} \mathrm{~s}^{-1}$ \\
Diffusivity other components & $10^{-9} \mathrm{~m}^{2} \mathrm{~s}^{-1}$ \\
{$[B]_{\mathrm{r}}$} & $1000 \mathrm{~mol} \mathrm{~m}^{-3}$ \\
{$[A]_{i}$} & $10 \mathrm{~mol} \mathrm{~m}{ }^{-3}$ \\
Equilibrium constant & $10^{-3} \leqslant K \leqslant 10^{4}$ \\
Ha-number & $0.10 \leqslant H a \leqslant 10^{6}$ \\
Solute loading & $\alpha=10^{-3}$
\end{tabular}


5. $\overline{5}$. Experimental verification

5.5.1. Introduction. The numerical models developed in the present study have been experimentally tested by means of the absorption of $\mathrm{CO}_{2}$ or $\mathrm{H}_{2} \mathrm{~S}$ into various aqueous alkanolamine solutions. Commercial grade amines of $98^{+} \%$ purity were used (Versteeg and van Swaaij, 1988b). The experiments were carried out at $298 \mathrm{~K}$ in a stirred vessel similar to the one used by Blauwhoff and van Swaaij (1985) under conditions that the gas liquid interfacc appeared to the cyc to be completely smooth and therefore was well defined. The absorption rates were determined for several gas-phase and liquid-phase mass transfer coefficients as a function of the gas-phase concentration $\quad\left(0.40 \mathrm{~mol} \mathrm{~m}^{-3} \leqslant\right.$ gas-phase concentration $\leqslant 41.0 \mathrm{~mol} \mathrm{~m}^{-3}$ ). In this way it was possible to study several kinetics regimes (e.g. slow reaction, fast reaction, instantaneous reaction and the intermediate regimes). Before the experiments could be simulated several assumptions had to be made in order to obtain enough information on the parameters which were necessary for the calculations.

The solubility of both $\mathrm{CO}_{2}$ and $\mathrm{H}_{2} \mathrm{~S}$ were obtained by means of the $\mathrm{CO}_{2}-\mathrm{N}_{2} \mathrm{O}$ analogy (Laddha et al., 1981) and for alkanolamines these solubilities were summarized by Versteeg and van Swaaij (1988a). A similar analogy was applied for the estimation of the diffusivity of the reactive solutes, and the actual value of both the diffusivity of the solute and the components already present in the liquid were calculated with a modified Stokes-Einstein relation (Versteeg and van Swaaij, 1988a). For the ionic products, the diffusivity has been given the same value in order to assure overall electroneutrality in the liquid and the values were taken equal to the component with the lowest diffusion coefficient.

Gas-phase and liquid-phase mass transfer coefficients of the stirred vessel were measured by means of physical absorption of high purity $\mathrm{N}_{2} \mathrm{O}$ into the solution for $k_{i}$, and by the absorption of very diluted $\mathrm{H}_{2} \mathrm{~S}$ into the aminc solution under conditions such that the mass transfer was gas phase controlled (see Danckwerts, 1970) for $k_{g}$, respectively. For the simulation the penetration theory has been applied because, according to Versteeg et al. (1987), for the experimental conditions used here this is the most realistic model.

The forward reaction between $\mathrm{H}_{2} \mathrm{~S}$ and the various alkanolamines was regarded as instantaneous with respect to mass transfer and has been approximated by very high $\left(\geqslant 10^{8}\right)$ reaction rate constants with $\gamma_{a}$ $=\gamma_{b}=\gamma_{c}=\gamma_{d}=1, m=\boldsymbol{n}=\boldsymbol{t}=\boldsymbol{v}=\mathbf{1}$ and $p=q=\boldsymbol{r}=\boldsymbol{s}=0$. The reaction rate constant for the reverse reaction was estimated by the assumption that at equilibrium forward and reverse reaction rates are equal, leading to:

$$
K=\frac{k_{m, n, p, q}}{k_{r, s, t, v}} .
$$

The forward reaction rate expression of $\mathrm{CO}_{2}$ with the various alkanolamines is rather complex (Blauwhoff $e t$ al., 1984) and therefore approximated by eq. (2). This approximation was allowed because the deviation of the approximate rate expression from the actual expression could be neglected for small variations of the amine concentrations, i.e. low solute loadings. The reverse reaction rate was calculated, similar as for $\mathrm{H}_{2} \mathrm{~S}$, according to eq. (40). The reaction constant of monoethanolamine (MEA) ([MEA] $=450$ mol m$~^{-3}$ ) was calculated according to Hikita et al. (1977) with $\gamma_{a}=\gamma_{c}=\gamma_{d}=1, \gamma_{b}=2, m=n=t=v=1, s=$ -1 and $p=q=r=0$ and for diethanolamine (DEA) ( $[\mathrm{DEA}]=2000 \mathrm{~mol} \mathrm{~m}^{-3}$ ) according to Blauwhoff et al. (1984), however, his results were reevaluated with improved data on the physical constants (Versteeg et al., 1987), with $\gamma_{a}=\gamma_{c}=\gamma_{d}=1, \gamma_{b}=2, m=n=t=v=1, s$ $=-1$ and $p=q=r=0$.

For the calculation of the equilibrium composition of the liquid the reader is referred to Blauwhoff and van Swaaij (1980).

5.5.2. $\mathrm{DEA}-\mathrm{H}_{2} \mathrm{O}$. The kinetic regimes studied were instantaneous reaction (for $\mathrm{H}_{2} \mathrm{~S}$ ) and fast reaction changing to instantaneous (for $\mathrm{CO}_{2}$ ). In Fig. 9 the measured absorption rates for two liquid-phase mass transfer coefficients, as a function of the gas-phase concentration, are presented together with the calculated rates for the absorption of $\mathrm{H}_{2} \mathrm{~S}$. From this figure it can be concluded that the model predicts the absorption rates of $\mathbf{H}_{2} \mathrm{~S}$ reasonably well. In $\mathrm{Fig}$. 10 the measured rates are compared with the calculated rates and besides the $\mathrm{H}_{2} \mathrm{~S}$ rates also the absorption rates for $\mathrm{CO}_{2}$ are presented. The model is able to predict all absorption rates with a maximum deviation of $20 \%$. However, for high experimental absorption rates a shift to somewhat higher experimental rates in comparison to the rates calculated was observed. For both

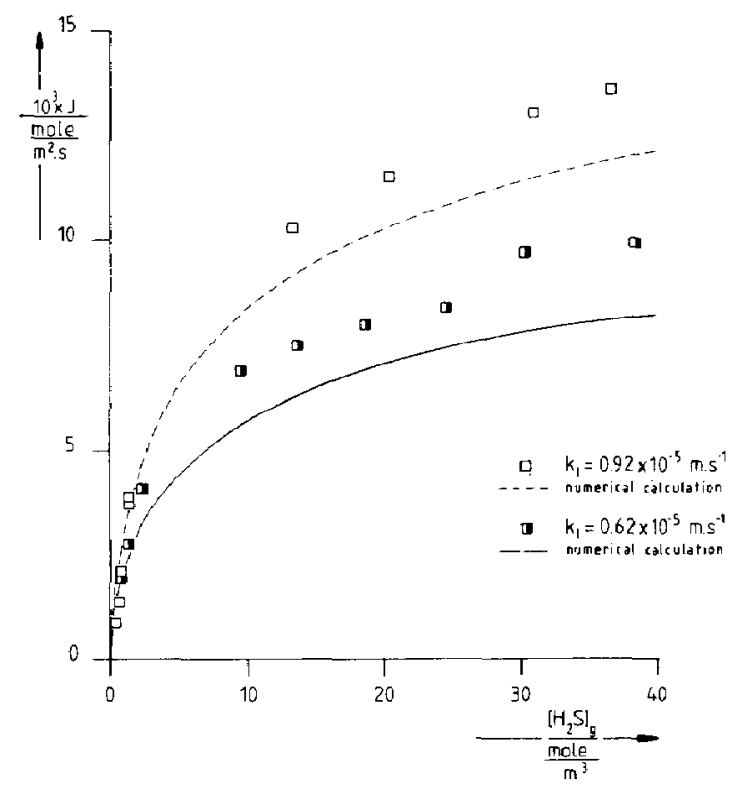

Fig. 9. Absorption rate of $\mathrm{H}_{2} \mathrm{~S}$ as a function of the gas-phase concentration in an aqueous DEA solution at $298 \mathrm{~K}$. 


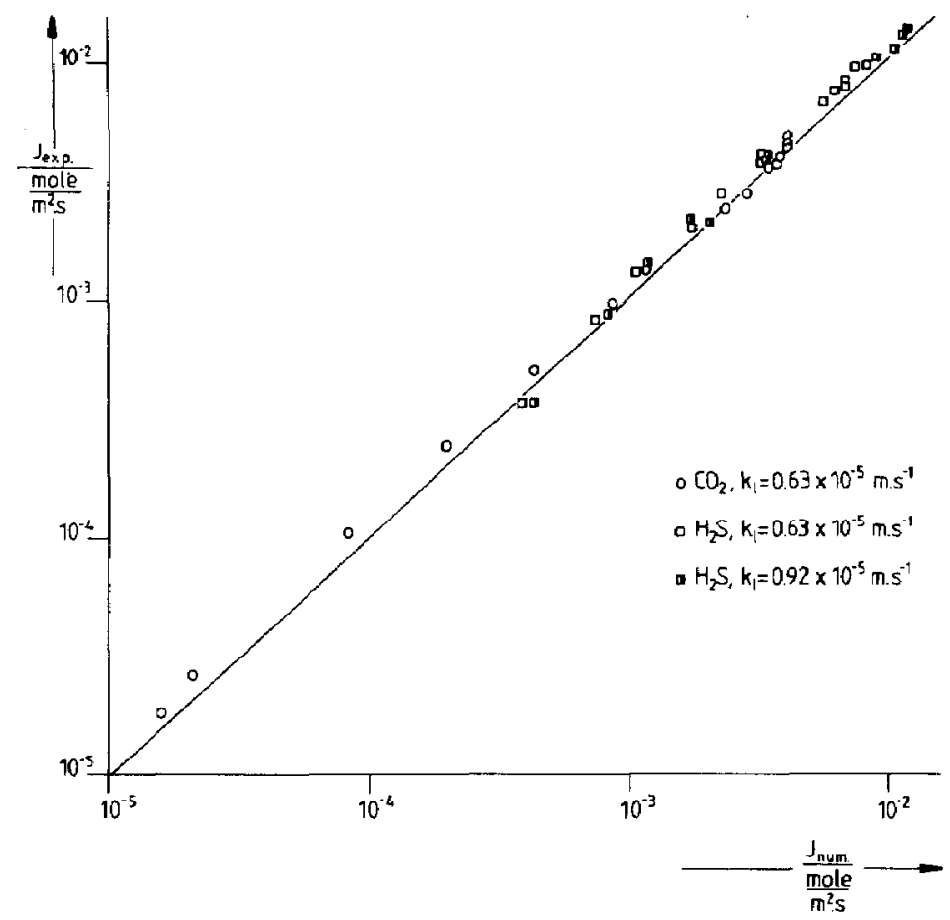

Fig. 10. Comparison between measured and calculated absorption rates of $\mathrm{H}_{2} \mathrm{~S}$ and $\mathrm{CO}_{2}$ in an aqueous DEA solution at $298 \mathrm{~K}$.

the absorption of $\mathrm{H}_{2} \mathrm{~S}$ and $\mathrm{CO}_{2}$ this increase is more pronounced for the instantaneous reaction regime and in this regime the reaction products are formed near the gas-liquid interface. As the products are ionic interfacial turbulence may occur and possibly the mass transfer rate was increased at this extreme condition by Marangoni effects.

5.5.3. $\mathrm{MEA}-\mathrm{H}_{2} \mathrm{O}$. For this system the instantaneous reaction regime was studied for $\mathrm{H}_{2} \mathrm{~S}$. For $\mathrm{CO}_{2}$ the absorption also occurred in the instantanenus reaction regime as can be calculated from the experimental conditions and the physico-chemical constants. However, it should be noted that the difference in reaction rates between both components is substantial. In Fig. 11 the results of the comparison are presented and for this system the measured absorption rates can be predicted with the model with a maximum deviation of $40 \%$.

For this system also an increasing deviation between the measured and calculated rates was observed for higher absorption rates. These deviations were much larger than for the system $\mathrm{DEA}-\mathrm{H}_{2} \mathrm{O}$ which may be attributed to the lower amine concentration and therefore to a relative higher increase of the concentration of ionic products near the gas-liquid interface.

5.5.4. Conclusions on the comparison with the experiments. Overall a good agreement is found over an extreme wide variation of the experimental conditions. The deviations between the experimental and the calculated rates may be induced by three effects:
Uncertainties in the physical constants necessary for the numerical calculation of the rates, especially the determination of the diffusion coefficients may lead to a deviation of the calculated absorption rate of about $10 \%$.

- The accuracy of the experimental method applied is about $10 \%$ which is due to low conversions of gas-phase concentration needed for the calculation of the absorption rates.

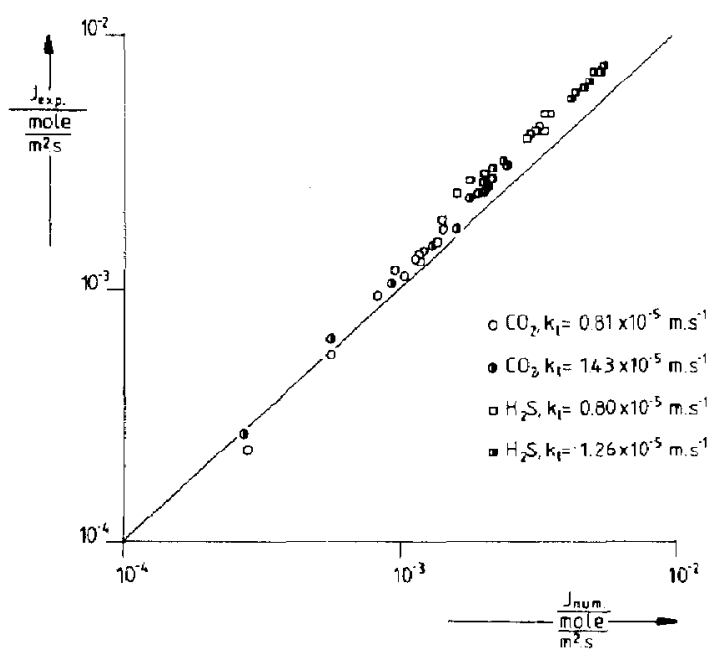

Fig. 11. Comparison between measured and calculated absorption rates of $\mathrm{H}_{2} \mathrm{~S}$ and $\mathrm{CO}_{2}$ in an aqueous MEA solution at $298 \mathrm{~K}$. 
-Marangoni effects at high absorption rates induced by high concentrations of ionic reaction products near the gas-liquid interface especially for the instantaneous reaction regime.

\section{CONCLUSIONS}

The absorption rates for the phenomenon mass transfer accompanied by a complex reversible reaction can be calculated over wide range of conditions with the numerical solution method presented in this study. From comparison of the numerical results with analytical asymptotic solutions it was concluded that the deviations could be neglected. Due to an additional transformation it was possible to improve the accuracy and minimise the computational time drastically. For reactions with "infinite" reaction rate constants it is possible to calculate the mass transfer rates with the present model in combination with the approximation of extremely high rate constants without a significant loss of accuracy.

The application of relations for the description of mass transfer accompanied by a chemical reaction derived for irreversible reactions cannot be applied to reversible systems. Even for systems with extremely low solute loadings the reversibility may have a substantial effect on the outcome of the calculations.

The approximate solutions for the phenomenon mass transfer followed by a chemical reaction presented in literature can be used to calculate the absorption rates for some specific situations but none of them can be applied generally and therefore are of restricted use. The linearization of the reaction term according to Hikita and Asai (1963) for reversible reactions as used by Onda et al. (1970) can lead to erroneous results and should always be used with care. Before the approximation can be applied it is necessary to check the approximation with the aid of a numerical solution. It seems interesting to develop an approximate solution for the film model according to the linearization technique presented by DeCoursey (1982).

Experimentally measured absorption rates in a stirred vessel with a flat interface of $\mathrm{H}_{2} \mathrm{~S}$ or $\mathrm{CO}_{2}$ in various aqueous alkanolamine solutions can be predicted fairly well with the numerically solved mass transfer model over a wide range of process conditions. However, for high absorption rates a substantial higher molar flux was measured than the calculated absorption rates which may be due to the occurrance of Marangoni effects, specifically for situations with relative high concentrations of ionic products near the gas-liquid interface.

Acknowledgements - These investigations were supported by the Technology Foundation, future Technical Science Branch of the Netherlands Organization for the Advancement of Pure Research (ZWO), and the Koninklijke/Shell Laboratorium Amsterdam. We also acknowledge $\mathrm{H}$. Bosch and $\mathbf{R}$. Heezen for their assistance in the experimental work and the numerical solution of the models.

\section{NOTATION}

$A$

$B$

C

$D$

$D_{\text {subserip }}$

d

E

$E_{\text {subseript }}$

$E_{\text {subseript, } \infty}$

F

$f_{1}$

$g$

$H a$

He

$J$

$j$

$K$

$k_{g}$

$k_{\text {! }}$

$k_{\text {subscript }}$

$K_{1}$

$l$

$M$

$m$

$m$

$N$

$n$

$p$

$p$

$p$
$q$

q

$r$

$r$

$s$

$t$

$t$

$v$

$w$

$\Delta w$

$x$

$z$

$\Delta z$

[ ]

\section{Greek letters}

$\alpha$

$\delta$ component $A$

component $B$

component $C$

component $D$

diffusivity, $\mathrm{m}^{2} \mathrm{~s}^{-1}$

ratio of diffusivities defined by eq. (30), 1

component $E$

enhancement factor defined by eq. (14) or (15), 1

infinite enhancement factor defined by cq. (39), 1

component $F$

function defined by DeCoursey (1982)

gas phase

Hatta-number defined by

$\left(k_{m, n, p, q}[A]^{m-1}[B]^{n}[C]^{p}[D]^{q} D_{a}\right)^{0.5} / k_{1}, 1$

dimensionless solubility defined as

$[A]_{1} /[A]_{\mathrm{g}}, 1$

molar flux, mol m-2 $\mathrm{s}^{-1}$

discrete time index

equilibrium constant, $\mathrm{mol}^{\left(-\tau_{a}-\tau_{b}+\tau_{c}+\tau_{d}\right)}$ $\mathrm{m}^{-3\left\{-\tau_{n}-\tau_{b}+\tau_{c}+\tau_{d}\right\}}$

gas-phase mass transfer coefficient, $\mathrm{m} \mathrm{s}^{-1}$ liquid-phase mass transfer coefficient, $\mathrm{ms}^{-1}$

reaction rate constant, $\mathbf{m}^{3(m+n+p+q-1)}$ $\mathrm{mol}^{-(m+n+p+q-l)} \mathrm{s}^{-1}$ or $\mathrm{m}^{3(\mathrm{r}+s+l+z-l)}$ $\mathrm{mol}^{-(r+s+t+v-l)} \mathrm{s}^{-1}$

constant used by DeCoursey (1982)

liquid phase

Hatta-number defined by DeCoursey (1982), 1

reaction order, 1

discrete space index

number of grid points

reaction order, 1

transformation parameter defined by eq. (16)

reaction order, 1

reaction order, 1

reaction rate, $\mathrm{mol} \mathrm{m} \mathrm{m}^{-3} \mathrm{~s}^{-1}$

place variable after transformation defined by eq. (26a), 1

reaction order, 1

reaction order 1

time variable, $s$

reaction order, 1

reaction order, 1

transformed time variable, $\mathrm{s}^{0.5}$

grid distance for time variable

dimensionless place variable, 1

transformed place variable, 1

grid distance for place variable.

liquid-phase concentration, $\mathrm{mol} \mathrm{m}^{-3}$

solute loading defined by $[A]_{*} /[B]_{i}, 1$

film thickness according to the film model, $m$ 


\section{Subscripts}

a

an

b

bulk

c

$d$

$\boldsymbol{t}$

$g$

$m$

$m$

num

0

$t$

\section{Superscripts}

0
1
$j$

derivative of function

initial value

first value

number indication of time step in discretization scheme

\section{REFERENCES}

Blauwhoff, P. M. M. and van Swaaij, W. P. M., 1980 Gas-liquid equilibria between $\mathrm{H}_{2} \mathrm{~S}, \mathrm{CO}_{2}$ and aqueous amine solutions. Proc. 2nd Int. Conf. on Phase Equilibria and Fluid Properties in the Chem Ind., EFCE Publication 11, Berlin, p. 78 .

Blauwhoff, P. M. M., Versteeg, G, F. and van Swaaij, W. P. M., 1984, A study on the reaction between $\mathrm{CO}_{2}$ and alkanolamines in aqueous solutions. Chem. Engng Sci. 39, 207-225

Blauwhoff, P. M. M. and van Swaaij, W. P. M., 1985 Simultaneous mass transfer of $\mathrm{H}_{2} \mathrm{~S}$ and $\mathrm{CO}_{2}$ with complex chemical reactions in an aqueous di-isopropanolamine solution. Chem. Engng Proc. 19, 67-83.

Cornelisse, R., Beenackers, A. A. C. M., van Beckum, F. P. H and van Swaaij, W. P. M., 1980, Numerical calculation of simultaneous mass transfer of two gases accompanied by complex reversible reactions. Chem. Engng Sci. 35 $1245-1260$
Danckwerts, P. V. and Kennedy, A. M., 1954, Kinetics of liquid-film process in gas absorption. Part 1: Models of the absorption process. Trans. Instn Chem. Engrs 32, s49-s53.

Danckwerts, P. V., 1970, Gas-Liquid Reactions. McGrawHill, New York.

DeCoursey, W. J., 1974, Absorption with chemical reaction: development of a new relation for the Danckwerts model. Chem. Engng Sci. 29, 1867-1872.

DeCoursey, W. J., 1982, Enhancement factors for gas absorption with reversible reaction. Chem. Engng Sci. 37 , 1483-1489.

Hikita, H. and $\Lambda$ sai, S., 1963, Gas absorption with $(m, n)$ thorder irreversible chemical reaction. Kagaku Kogaku 11 $823-830$.

Hikita, H., Asai, S., Ishikawa, H. and Honda, M, 1977, The kinetics of reactions of carbon dioxide with monocthanol amine, diethanolamine and triethanolamine by a rapid mixing method. Chem. Engng J. 13, 7-12

Hikita, H., Asai, S., Yano, A. and Nose, H., 1982, Kinetics of absorption of carbon dioxide into aqueous sodium sulfite solutions. A.I.Ch.E. J. 28, 1009-1015.

Huang, C.-J. and Kuo, C.-H., 1965, Mathematical models for mass transfer accompanied by reversible chemical reaction A.I.Ch.E. J. 11, $901-910$.

van Krevelen, D. W. and Hofijzer, P. J., 1948, Kinetics of gas-liquid reactions. Part 1: General theory. Rec. Trav. Chim. 67, 563-586.

Laduha, S. S., Diaz, J. M. and Danckwerts, P. V., 1981, The $\mathrm{N}_{2} \mathrm{O}$ analogy: the solubilities of $\mathrm{CO}_{2}$ and $\mathrm{N}_{2} \mathrm{O}$ in aqueous solutions of organic compounds. Chem. Enging Sci. 36, $229-230$

Olander, D. R., 1960, Simultaneous mass transfer and equilibrium chemical reaction. A.I.Ch.E. J. 6, 233-239.

Onda, K., Sada, E., Kobayashi, T. and Fujine, M, 1970, Gas absorption accompanied with complex chemical reactions-I. Reversible chemical reactions. Chem. Engng Sci. 25, $753-760$

Onda, K., Sada, E., Kobayashi, T. and Fujine, M., 1972, Gas absorption accompanied by complex chemical reactions IV. Unsteady state. Chem. Engng Sci. 27, 247-255.

Perry, R. H. and Pigford, R. L., 1953, Kinetics of gas-liquid reactions. Simultancous absorption and chemical reaction. Ind. Engng Chem. 45, 1247-1253.

Secor, R. M. and Beutler, J. A., 1967, Penetration theory for diffusion accompanied by a reversible chemical reaction with generalized kinetics. A.I.Ch.E. J. 13, 365-373.

Sherwood, T. K. and Pigford, R. L., 1952, Absorption and Extraction, p. 332. McGraw-Hill, New York.

Versteeg, G. F., Blauwhoff, P. M. M. and van Swaaij, W. P. M., 1987, The effect of diffusivity on gas - liquid mass transfer in stirred vessels. Experiments at atmospheric and elevated pressures. Chem. Engng Sci. 42, 1103-1119.

Versteeg, G. F. and van Swaaij, W. P. M., 1988a, The solubility and diffusivity of acid gases in aqueous amine solutions. J. Chem. Engng Data 32, 29-34.

Versteeg, G. F. and van Swaaij, W. P. M., 1988b, On the kinetics between $\mathrm{CO}_{2}$ and alkanolamines both in aqueous and non-aqueous solutions-I. Primary and secondary amines. Chem. Engng Sci. 43, 573-585. 